Ann. Zootech., r978, 27 (3), 363-376.

\title{
Comparaison chez le mouton, de l'oxyde de chrome-papier et du radiocérium, marqueurs de la phase solide des digesta
}

\author{
Nicole THILL, E. FRANÇOIS $\left({ }^{*}\right)$, A. THEWIS $\left({ }^{* *}\right)$ et Marie-France THIELEMANS (*) \\ avec la collaboration technique de \\ P. Bosch, A. Colinet, F. Dupont (*), J. C. Pichon et M. Pichon \\ Station de Zootechnic, \\ $\left(^{*}\right)$ Station de Chimie et de Physique agricole, Centves de Recherches agronomiques de l'Etat, \\ IT 5, chaussée de Wavre, $B 58$ oo Gembloux (Belgique) \\ (**) Chaire de Physiologie animale et de Zootechnie, \\ Faculté des Sciences agronomiques de l'État, \\ 2, passage des Déportés, B 5800 Gembloux (Belgique)
}

\section{Résumé}

L'étude comparée, chez le Mouton alimenté au foin de prairie haché, de l'excrétion fécale et du transit dans le tube digestif de l'oxyde de chrome-papier $\left(\mathrm{Cr}_{2} \mathrm{O}_{3}\right)$ et du radiocérium ( ${ }^{144} \mathrm{Ce}$ ), a permis de montrer que le comportement des deux indicateurs est pratiquement identique à ce point de vue.

I,es taux de récupération fécale observés pour le $\mathrm{Cr}_{2} \mathrm{O}_{3}$, pratiquement quantitatifs, confirment le caractère non absorbable, souvent remis en question, de cet indicateur. La forme d'administration de l'oxyde de chrome-papier mise au point — il est incorporé à du foin de luzerne mis en pellets à la presse à filière - assure, sans perturber l'animal, une ingestion quantitative de l'indicateur. Le $\mathrm{Cr}_{2} \mathrm{O}_{3}$ a été administré, sous cette forme, aux 6 moutons utilisés. Le radiocérium a été offert avec la ration de foin de prairie haché intégralement et uniformément marqué, au premier groupe de 3 moutons, sous la forme de pellets apportant les deux indicateurs, au second groupe.

Les résultats obtenus semblent accroître les perspectives d'emploi de l'oxyde de chromepapier offert sous la forme décrite.

\section{I. - Introduction}

Comme le fait remarquer von ENGELHARDT (I974), aucun des marqueurs digestifs disponibles actuellement ne peut être considéré comme parfait.

La condition première à remplir est de ne pas être absorbés dans le tube digestif : ils doivent être récupérés intégralement dans les matières fécales. Cette 
caractéristique est exigée tant pour les études de digestibilité que pour celles visant à déterminer l'ingestion au pâturage.

En outre, ils doivent rester associés aux constituants de la phase marquée, au cours du transit. Cette exigence est particulièrement importante pour les études de digestion menées sur des animaux munis de fistules simples.

L'oxyde de chrome proposé par EDIN (I9I8) comme indicateur inerte a été fort utilisé, principalement en vue de la détermination de digestibilité des rations par de nombreux auteurs, pour de nombreuses espèces animales (SCHURCH, LLOxD et Crampton, i95o; Kane, Jacobson et Moore, I950; Clawson et al., r955; MOORE, I957).

Si l'indicateur administré est récupéré intégralement dans certains cas (KANE, et al., I950; Clawson et al., I955; Davis, ByER et LUBER, I958; EIAM et al., I962), dans de nombreux autres, les résultats sont décevants (CRAMPTON et LLOYD, I95I; CoRbetT et al., I958; HARdison et al., I959; CLANTON, I962; LASSITER, AliIGOOD et McGaughey, ig66; Macguire, Bradiey et LitTle, ig66; Curran, LEaver et Weston, I967; KNAPKA et al., I967; PHAR et al., I970).

Pour rendre compte de la récupération incomplète, CRAMPTON et LLOYD (I95I) émettent 1'hypothèse d'une rétention partielle dans le tube digestif, et DEINUM, IMMINk et DEIJs (I962) pensent qu'une certaine absorption de l'oxyde de chrome n'est pas à exclure. De plus, CURRAN, LEAVER et WESTON (I967) font remarquer qu'une régurgitation d'une partie de l'indicateur est toujours possible.

Toutefois, UTLEY et al. (I970), après administration d'une dose unique de ${ }^{51} \mathrm{Cr}_{2} \mathrm{O}_{3}$ chez les bovins ne décèlent aucune trace du radiochron:e dans le sang et 1'urine, ce qui semblerait confirmer le caractère non absorbable de l'oxyde de chrome; il ne récupèrent, cependant, que $87,2 \mathrm{p}$. roo de cette dose dans les fèces, après 9 jours de récolte.

Ce rapide aperçu de la bibliographie nous montre que les opinions sont fort partagées quant au caractère non absorbable de l'oxyde de chrome.

D'autre part, nous l'avons vu, pour étudier les aspects quantitatifs de la digestion, les indicateurs employés doivent non seulement être strictement non absorbables, mais ils doivent en outre accompagner les constituants solides de la ration dans leur transit.

Les radiolanthanides et le radiocérium en particulier, satisfont parfaitement ces exigences (ELLIS, I 968; EiLlis et HuSTON, I968) et sont de plus en plus utilisés (FRANÇOIS, " COMPÈre et RONDIA, I968; FRANÇOIS et COMPËRE, I97I et I972; Grovum et Hecker, I973; Grovum et Williams, I973; Poncet, I976). L'absorption du complexe ruthénium-phénanthroline marqué au ${ }^{103} \mathrm{Ru}$ proposé par TAN, WESTON et HoGAN (I97I), fortement fixé aux particules alimentaires, est faible. La période $d u{ }^{103} \mathrm{Ru}$ en réduit, selon ces auteurs, les risques d'emploi. Cependant, l'emploi de ces marqueurs radioactifs est freiné par suite des risques de contamination et limité à des locaux parfaitement aménagés et contrôlés.

Dans ce contexte, et tenant compte du fait que $\mathrm{Cr}_{2} \mathrm{O}_{3}$ est utilisé par plusieurs équipes de chercheurs étudiant les phénomènes de la digestion (MACRAE et UlYATT, I972; VÉRITÉ et al., I977), nous avons voulu réévaluer ses caractéristiques essentielles.

Si l'oxyde de chrome en poudre, même intimement mélangé à la ration est excrété très irrégulièrement (ELAM, PUTNAM et DAVIS, I959), et en tout cas, plus irrégulièrement que le ${ }^{144} \mathrm{Ce}$ (Hustow et ELLIS, I968), l'emploi de papier imprégné de $\mathrm{Cr}_{2} \mathrm{O}_{3}(\mathbf{1})$, semble représenter une nette amélioration à cet égard (CORBETT et al., 
ig60; Cowlishaw et Alder, ig63; Troelsex, Ig66; Langlands et al., ig63). Ce support améliore vraisemblablement le mélange du marqueur et du contenu du rumen.

L'emploi de cette préparation ne pouvait cependant être envisagé que si on parvenait à la faire accepter aisément et intégralement par l'animal. Nous y sommes parvenus en incorporant les morceaux de papier imprégné de $\mathrm{Cr}_{2} \mathrm{O}_{3}$ à des pellets de luzerne.

Nous disposions dès lors d'un mode d'administration pratique se prêtant particulièrement bien aux études de consommation à l'herbage, par exemple.

L'étude de la bibliographie montre que les conditions d'administration - mode et fréquence - influencent très fortement les résultats obtenus avec $\mathrm{Cr}_{2} \mathrm{O}_{3}$. Nous avons donc décidé d'étudier son comportement et ses propriétés en fonction de ces conditions nouvelles d'emploi.

L'étude de la migration comparée de $\mathrm{Cr}_{2} \mathrm{O}_{3}$ et de ${ }^{{ }^{144}} \mathrm{Ce}$ dans le tube digestif du Mouton - le second marqueur étant strictement non absorbé et fortement lié aux particules de digesta — se prête particulièrement à ce but et constitue le sujet du présent travail.

\section{II. - Matériel expérimental et techniques utilisées}

\section{Animaux et alimentation}

Les animaux utilisés sont des béliers de race Texel d'un poids moven de $40 \mathrm{~kg}$, adultes, régulièrement traités contre les parasites. Ils sont alimentés au foin de prairie, en bergerie, d'abord. En vue de l'étude comparée de la cinétique d'excrétion de $\mathrm{Cr}_{2} \mathrm{O}_{3}$ et de ${ }^{\mathbf{1 4 4}} \mathrm{Ce}$, ils sont placés en cage à métabolisme, où le même foin, haché, est offert deux fois par jour, à 9 et $I 6$ h. Pour les sujets 62,57 et 55 , il a été, au préalable, marqué uniformément au radiocérium (FRAnçoIs et THEwIs, I976). L'oxyde de chrome est administré sous la forme de pellets, simplement marqués pour les moutons 62,57 et 55 ; doublement $-\mathrm{Cr}_{2} \mathrm{O}_{3}$ et ${ }^{144} \mathrm{Ce}-$ pour les moutons 53,6 et 60 (voir ci-dessous). Les pellets sont distribués en deux doses exactement pesées, de quelque $25 \mathrm{~g}$, apportant chacune $2 \mathrm{~g}$ de $\mathrm{Cr}_{2} \mathrm{O}_{3}$ avant les distributions de foin. Ils sont immédiatement et intégralement consommés. L'absence de pertes a pu être contrôlée, chaque fois, pour les pellets radioactifs, avec un simple détecteur de radiations. L'indice d'ingestion (1) moyen des animaux est de 29 .

\section{Préparation des aliments marqués}

La distribution des indicateurs débute 7 jours avant les premières récoltes de fèces, laps de temps amplement suffisant pour réaliser l'équilibre de marquage.

\section{L'oxyde de chrome-papier}

Les lanières de papier imprégné de $\mathrm{Cr}_{2} \mathrm{O}_{3}$ du Rowett Institute sont hachées en morceaux de quelques millimètres; ceux-ci sont ensuite mélangés à la farine de luzerne.

(1) Indice d'ingestion $\mathrm{I}=\frac{\text { quantité de MS ingérée en } \mathrm{g}}{\text { poids de l'animal en } \mathrm{kg}}$ 
Une concentration dans la matière sèche de $7 \mathrm{p}$. roo permet encore une bonne répartition de l'indicateur dans la masse. Ise passage du mélange dans une presse à filière ${ }^{(1)}$ de laboratoire donne des pellets bien formés ne se délitant pas et facilement acceptés par les animaux. Les particules de petites dimensions sont éliminées par tamisage afin d'assurer une ingestion quantitative des doses de marqueurs. La presse utilisée permet de fabriquer une cinquantaine de kilogrammes de pellets en une journée. L'indicateur a été distribué sous cette forme à tous les sujets.

Le radiocérium est distribué sotıs 2 formes : foin uniformément marqué et pellets de luzerne. Le marquage de la masse de foin consommé par un animal pendant la durée de la période expérimentale est réalisé suivant la technique décrite par FRANçoIs et THEWIs (I976). Ce foin est identique à celui - non radioactif offert aux sujets 53,6 et 60 , recevant le radiocérium incorporé aux pellets de luzerne. Ces derniers sont préparés suivant la technique décrite ci-dessus : le papier à l'oxyde de chrome est traité préalablement au mélange et au pressage au moven d'une solution de radiocérium projetée par nébulisation.

\section{Récolte et traitement des fèces}

Les fèces sont récoltées quantitativement, journellement d'abord pendant 3 jours, puis toutes les $2 \mathrm{~h}$ pendant $48 \mathrm{~h}$, puis de nouveau une fois par jour pendant 2 jours. Elles sont séchées dans leur totalité à $100{ }^{\circ} \mathrm{C}$ jusqu'à poids constant, mou lues, échantillonnées $\in t$ sotumises à l'analyse.

\section{Abattage des animaux et traitement des contenus du tube digestif}

Les sujets sont sacrifiés à jeun entre 8 et $9 \mathrm{~h}$ du matin. Ils sont abattus au révolver et saignés complètement. Le contenu du réticulo-rumen est soigneusement échantillonné : des prélèvements sont effectués en divers endroits de la masse et traités séparément. Le reste du tube digestif est traité suivant la technique habituelle (THEwis et al., I976).

\section{Déterminations analytiques}

\section{Radiochimiques}

Le couple de radionuclides en filiation ${ }^{144} \mathrm{Ce}-{ }^{144} \mathrm{Pr}$, cérium-praséodyme, est dosé par son émission bêta à l'aide d'un détecteur à courant gazeux, à fenêtre mince et à faible mouvement propre (I CPM) $\left({ }^{2}\right)$.

Les échantillons destinés au comptage sont obtenus par pressage hydraulique de la matière séchée et moulue (voir ci-dessous), suivant la technique décrite par THEWIS, FrançoIs et THILL (I975).

Deux modifications, cependant : les fèces sont additionnées de caséine en poudre à raison de $0,5 \mathrm{~g}$ pour $4,5 \mathrm{~g}$ de matière et de quelques gouttes d'eau et on homogénéise parfaitement avant pressage; ceci, afin d'obtenir des disques parfaitement formés et résistants.

De plus, $0,5 \mathrm{~g}$ de contenu, séché et moulu également, sont additionnés de $4 \mathrm{~g}$

(1) California, modèle CL 3.

(2) Nuclear Chicago Searle. 
de matière fécale non radioactive et de $0,5 \mathrm{~g}$ de caséine, afin d'uniformiser au maximum la structure physique en vue des comptages bêta. Les échantillons de fèces et d'aliment qui doivent être comparés aux contenus sont préparés bien entendu de la même manière, par dilution avec de la matière fécale non radioactive.

\section{Chimiques}

I'oxyde de chrome est déterminé suivant une méthode rapide et reproductible développée par Françors, ThILL et ThEwis, I978. L'échantillon - I $g$ de matièreest attaqué par le mélange nitroperchlorique dans un appareil approprié (pour éliminer tout risque d'explosion des perchlorates et de volatilisation du chlorure de chromyle) jusqu'à oxydation complète du $\mathrm{Cr}_{2} \mathrm{O}_{3}$ en $\mathrm{Cr}^{6+}$.

Une courte ébullition en présence de persulfate réoxyde la totalité du chrome (dont une part importante se réduit spontanément au cours du refroidissement du résidu de minéralisation). Le bichromate peut ensuite être titré directement par le sel de Mohr, en présence de diméthylamine sulfonée, comme indicateur, ou mieux, au titrateur automatique (électrodes Pt-calomel).

\section{III. - Résultats}

\section{Excrétion fécale du $\mathrm{Cr}_{2} \mathrm{O}_{3}$ et taux de récupération}

L'évolution de la concentration en indicateur dans les fèces est donnée à titre d'exemple aux figures I et 2, pour les animaux 57 et 60 , ayant reçu, le premier, du foin marqué au radiocérium et des pellets de luzerne à l'oxyde de chrome-papier

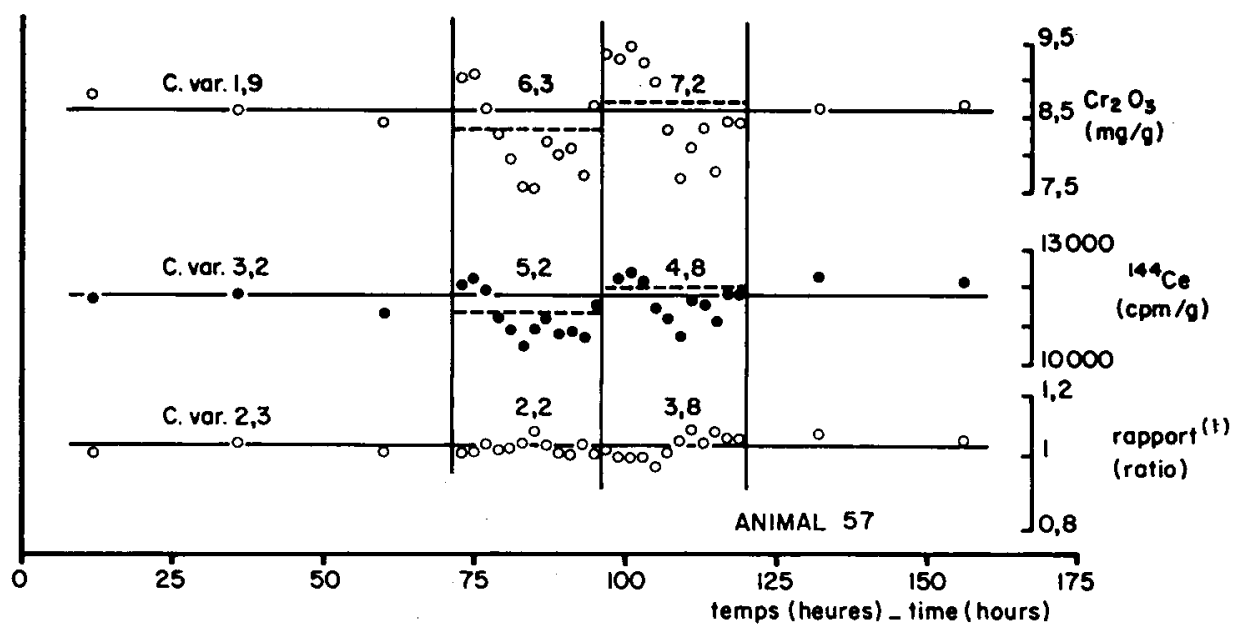

FIG. I. -- Excrétion fécale du vadiocérium et de l'oxyde de chrome administrés respectivement sous la forme de foin marqué uniformément et de pellets contenant du papier imprégné. Faecal excretion of radiocerium and chromium sesquioxide administered in form of labelled chopped hay and lucerne pellets incorporating the chromium oxide-paper respectively

(1) Il s'agit du rapport de la concentration en ${ }^{144} \mathrm{Ce}$ à celle en $\mathrm{Cr}_{2} \mathrm{O}_{3}$. $\mathrm{I}_{\mathbf{r}}$ a valeur correspondant à la première observation a été prise comme unité (Ratio of radiocerium to chromium sesquioxide concentration. The first observation has been taken as unity). 
et le second, des pellets de luzerne apportant les deux indicateurs. Les temps sont comptés à partir du début de la première récolte journalière. Ces deuxrexemples nous montrent que les schémas d'excrétion des deux indicateurs sont fort comparables, pour les deux modes d'administration du ${ }^{144} \mathrm{Ce}$. Le rapport $\mathrm{R}$ met cependant en évidence une légère différence de comportement, une certaine périodicité apparaît en effet dans son évolution. Le tableau I confirme les constatations ci-dessus. I1 reprend sous une forme condensée les coefficients de variation caractérisant l'évolution de la concentration fécale pour les deux indicateurs et pour tous les sujets pour les périodes de $2 \mathrm{~h}$ et d'un jour.

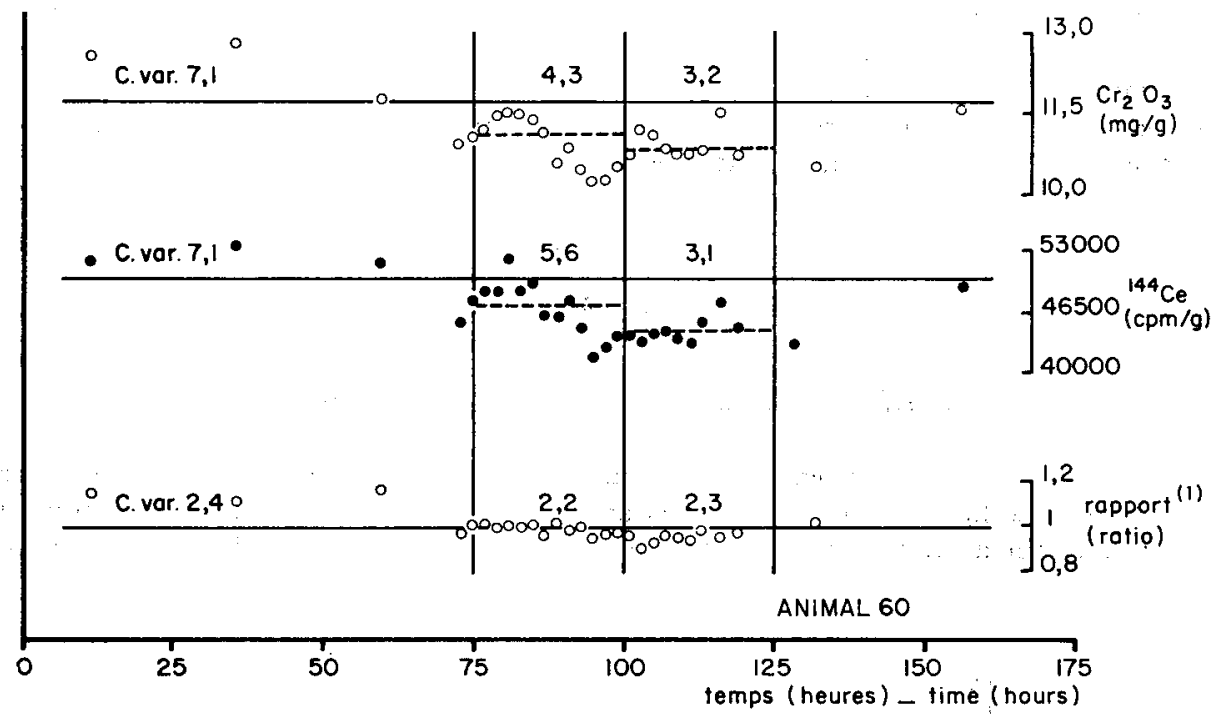

FIG. 2. - Excrétion fécale du radiocévium et de l'oxyde de chrome administrés sous la forme de pellets doublement marqués.

Faecal excretion of radiocerium and chromium sesquioxide administered in form of double labelled pellets.

(1) Il s'agit du rapport de la concentration en ${ }^{144} \mathrm{Ce}$ à celle en $\mathrm{Cr}_{2} \mathrm{O}_{3}$. I a valeur correspondante à la première observation a été prise comme unité (Ratio of radiocerium to chromium sesquioxide concentration. The first observation has been taken as unity).

Pour le sujet 55, une erreur dans l'administration des indicateurs ne nous permet pas de donner des valeurs antérieures à $1 \mathrm{a} I 3^{2} \mathrm{~h}$ de récolte.

On constate en outre, que le mode d'administration employé pour l'oxyde de chrome - faible fraction de la ration, offerte en deux fois et consommée très rapidement - ne produit guère plus de variations dans la concentration fécale que le mode pratiquement continu, optimal, utilisé pour le ${ }^{144} \mathrm{Ce}$.

Les taux de récupération des deux indicateurs sont donnés au tableau 2 .

Le bilan des indicateurs n'a pu être établi pour le sujet 53 . On remarque que les valeurs observées sont, dans l'ensemble, très proches de Ioo p. Ioo. Le sujet 57 présente une anomalie. L'excès observé pour les deux indicateurs est du même ordre de grandeur. 


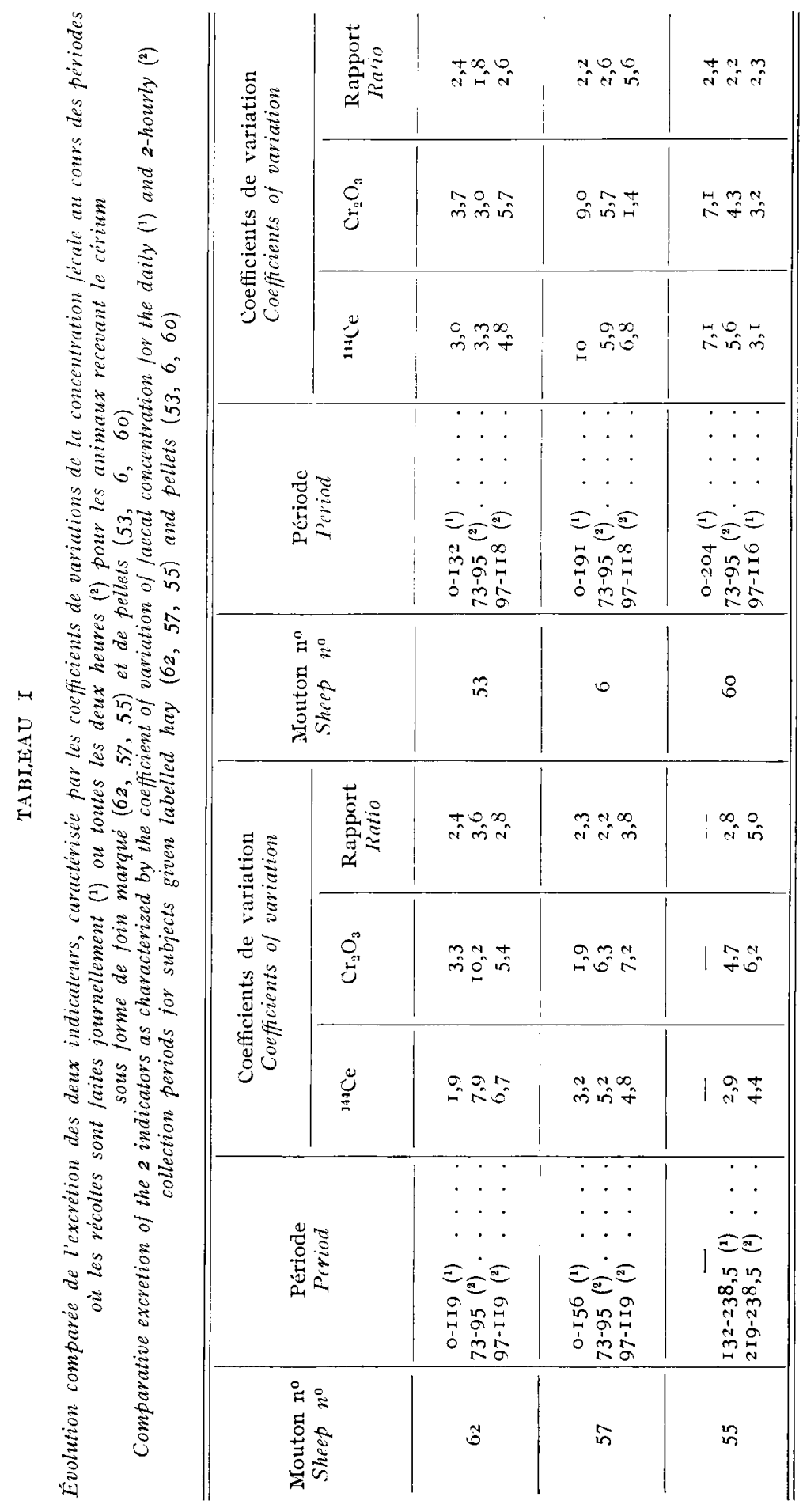




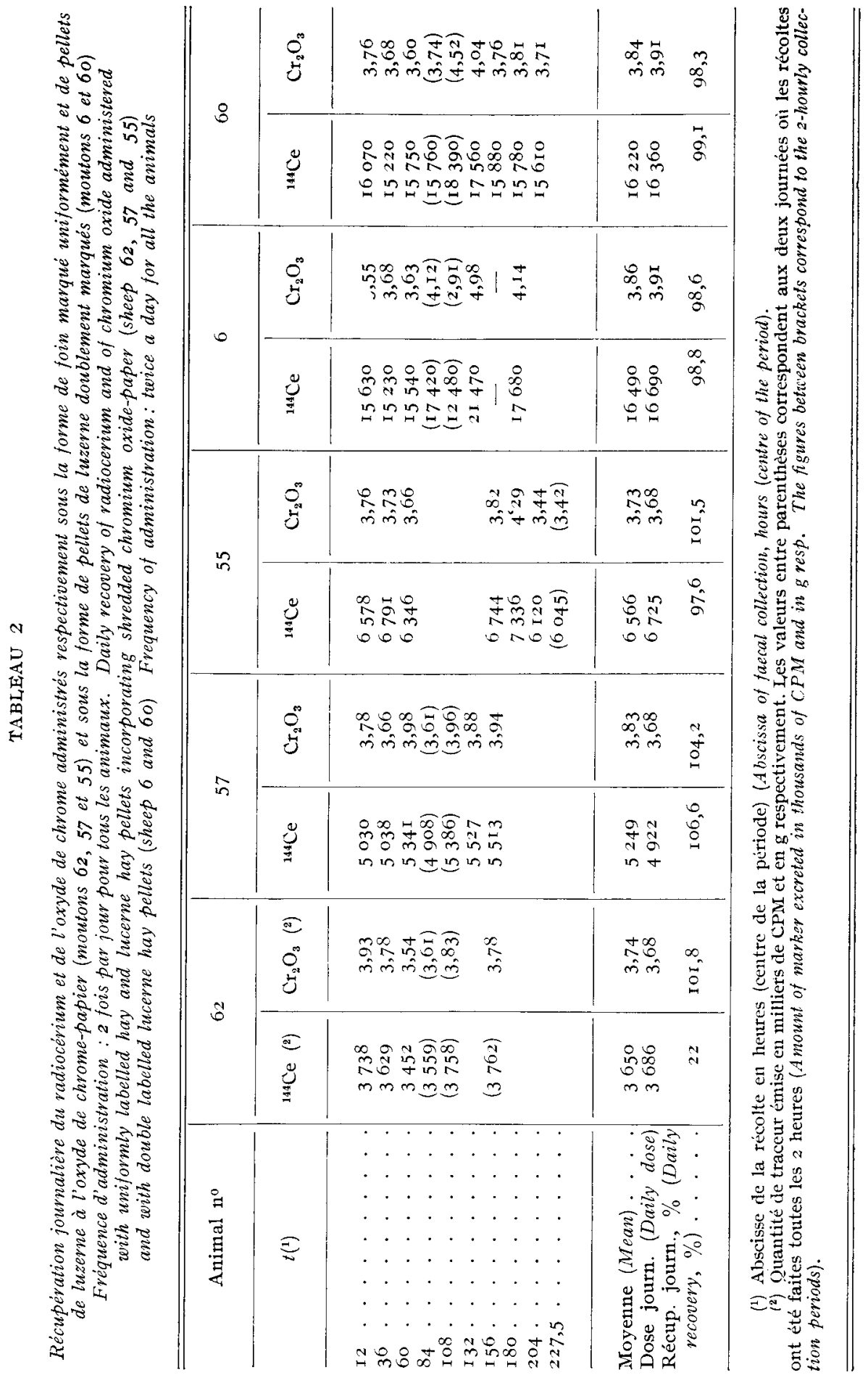




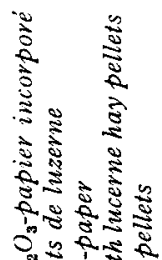
डे

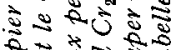

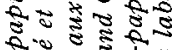
उ $\approx$ 究 $\approx$ จे

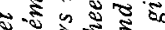

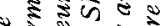
월 कू है

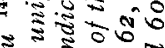

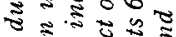
$m=2.0$ 0 os 50 \& 5 . क

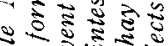

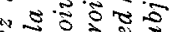
$\approx$ का

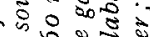
2क

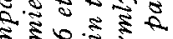

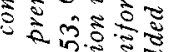
- 2 क ज

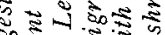

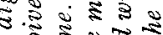
엉

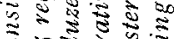

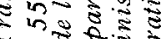
व है है in 0 己 वि है. $\cong$

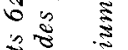

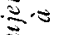
: $\stackrel{3}{4}$

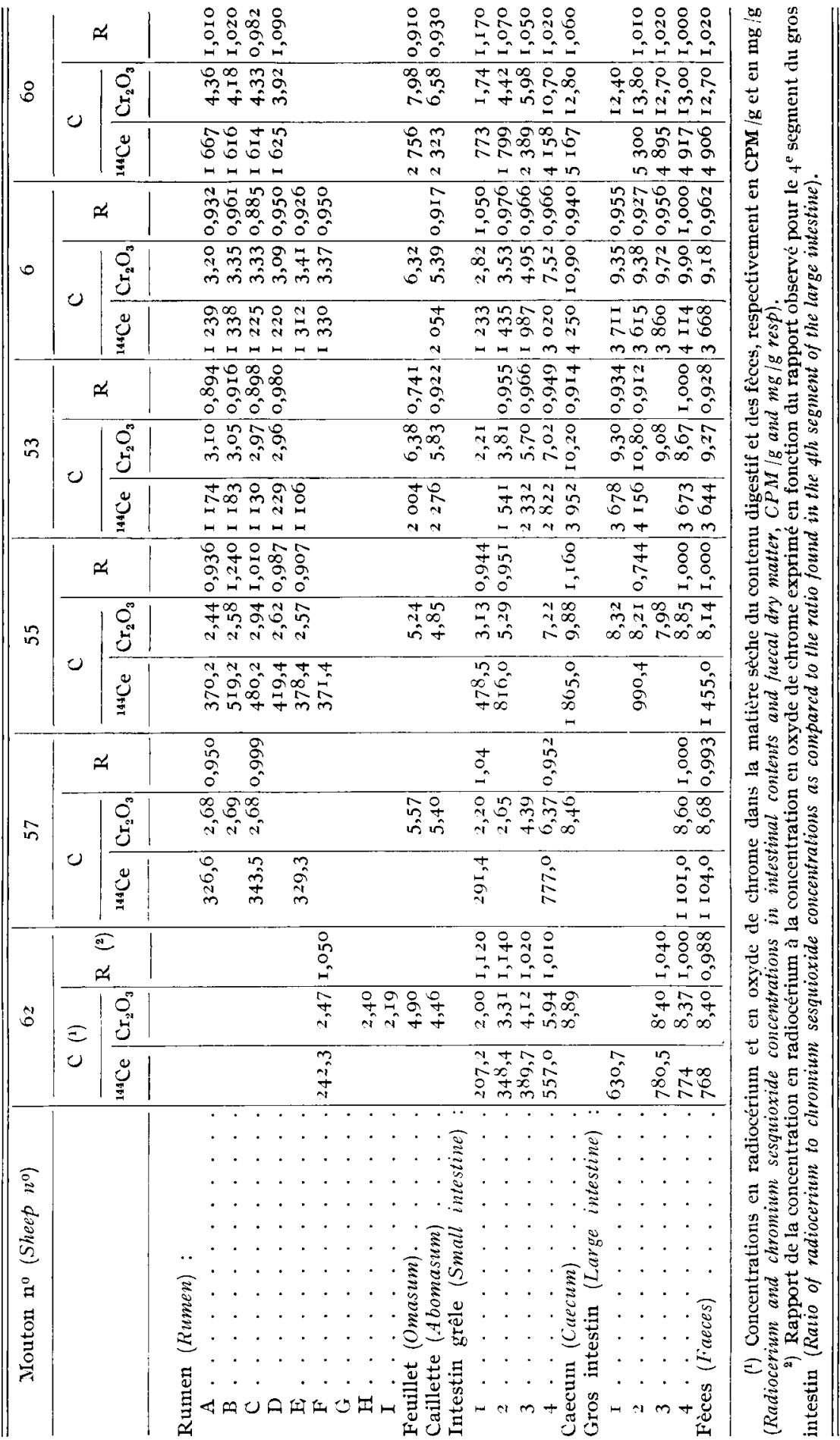




\section{Transit comparé des deux marqueurs dans le tube digestif}

L'évolution de la concentration en ${ }^{144} \mathrm{Ce}$ et en $\mathrm{Cr}_{2} \mathrm{O}_{3}$ dans les divers réservoirs et segments du tube digestif ainsi que dans les fèces émises immédiatement avant l'abattage est donnée aut tableau 3 .

Le rapport des deux traceurs dans chaque segment est également donné el, fonction de celui observé pour le dernier segment du gros intestin.

Rappelons que la concentration en ${ }^{144} \mathrm{Ce}$ des divers contenus a été déterminée après dilution avec de la matière non radioactive; ce qui explique que les valeur: notées pour les fèces émises avant abattage pour les sujets 57 et 60 sont nettement plus faibles qu'au tableau 2 .

Un certain nombre d'échantillons manquent pour les sujets 62,57 et 55 ; après prélèvement des échantillons nécessaires à une autre étude menée simultanément sur ces mêmes sujets, nous ne disposions plus d'assez de matière pour le dosage.

On remarque la bonne répartition des deux indicateurs dans le contenu du réticulo-rumen des moutons 53,6 et 60 . Les données des autres moutons recevant les indicateurs sous deux formes différentes sont fragmentaires à ce niveau, constatons néanmoins que le radiocérium bien réparti chez l'animal 57 , l'est moins bien chez le 55 .

Enstuite, si la concentration en ${ }^{144} \mathrm{Ce}$ et en $\mathrm{Cr}_{2} \mathrm{O}_{3}$ augmente irrégulièrement par suite de la digesto:1, leur rapport reste remarquablement constant dans l'ensemble et ce, pour les deux modes d'administration.

\section{Discussion et conclusion}

Les taux de récupération obtenus pour $\mathrm{Cr}_{2} \mathrm{O}_{3}$ nous paraissent prouver définitivement son carastère non absorbable. Ie fait que les légers écarts observés pour $\mathrm{Cr}_{2} \mathrm{O}_{3}$ sont du même ordre de grandeur que pour ${ }^{144} \mathrm{Ce}$, dont le caractère non absorbable a été maintes fois vérifié, indique qu'ils sont le résultat d'erreurs accidentelles. Cette observation confirme 1'opinion de PE'TRY et ENDERs (I 974), fondée sur les propriétés chimiques de $\mathrm{Cr}_{2} \mathrm{O}_{3}$ dont l'insolubilité et l'extrême résistance aux agents acides oxydants rendent fort invraisemblable l'hypothèse d'une absorption.

La constance du rapport de la concentration des deux indicateurs dans tout le tractus digestif apporte une preuve supplémentaire du caractère non absorbable de $\mathrm{Cr}_{2} \mathrm{O}_{3}$. Notons que la légère diminution de ce rapport dans le feuillet n'intéresse qu'une faible partie du contenu digestif.

Par ailleurs, l'étude comparée des courbes d'excrétion fécale des deux indicateurs montre que le $\mathrm{Cr}_{2} \mathrm{O}_{3}$ - administré sous la forme de pellets de luzerne contenant des morceaux de papier imprégné - a un comportement global fort comparable à celui du radiocérium dans le tube digestif du Mouton.

Notons que Huston et Eiris (I 968) obtiennent des résultats nettement moins bons pour le $\mathrm{Cr}_{2} \mathrm{O}_{3}$ en poudre que pour le ${ }^{144} \mathrm{Ce}$ avec des moutons recevant ces deux marqueurs répartis dans du foin de luzerne moulu. Ils pensent que cela serait dî à une migration séparée du $\mathrm{Cr}_{2} \mathrm{O}_{3}$ et de la matière sèche des digesta. Dans leurs essais, 1e $\mathrm{Cr}_{2} \mathrm{O}_{3}$, prémélangé à $\mathrm{I} \mathrm{kg}$ de foin de luzerne broyé, a été réparti dans une masse de $45 \mathrm{~kg}$ de ce même foin broyé à l'aide d'un mélangeur mécanique. Il faut remarquer qu'il est difficile de réaliser un mélange homogène et stable de $\mathrm{Cr}_{2} \mathrm{O}_{3} \mathrm{sec}$, 
produit de densité élevée et d'une poudre végétale. On ne peut, à notre avis, exclure l'influence de telles conditions de mélange sur les résultats obtenus.

Quant aux fluctuations observées dans la concentration fécale, le fait qu'elles sont du même ordre de grandeur pour les deux indicateurs, même administrés différemment — ${ }^{144} \mathrm{Ce}$ d'une façon continue et le $\mathrm{Cr}_{2} \mathrm{O}_{3}$ en deux fois - montre qu'elles sont liées aux processus digestifs; une augmentation de la fréquence d'administration des pellets à l'oxyde de chrome-papier ne pourrait guère les réduire, dans le cas présent.

Notons que, pour le radiocérium, les coefficients de variation de la concentration fécale sont fort comparables à ceux observés par PONCE'T (I976) sur des moutons recevant $z$ fois par jour le marqueur fixé sur loo g de foin.

La bonne répartition relative des deux marqueurs dans le contenu du rumen à l'abattage pour les sujets recevant du foin uniformément marqué au ${ }^{\mathbf{1 4}} \mathrm{Ce}$ et des pellets au $\mathrm{Cr}_{2} \mathrm{O}_{3}$-papier confirme la valeur du mode d'administration mis au point et l'importance de ce facteur sur la qualité du marquage obtenu. La libération progressive du $\mathrm{Cr}_{2} \mathrm{O}_{3}$ au cours de la digestion du papier par la microflore du rumen et la division des languettes avant le conditionnement en pellets, constituent sans doute les principales raisons de cette réussite.

Dans les conditions de nos expériences, aucune concentration locale due à une éventuelle sédimentation de ce marqueur n'a été observée dans le contenu du rumen. L'oxyde de chrome semblerait s'associer aux particules de digesta dès sa libération et les accompagner dans leur transit. Le fait que les deux indicateurs transitent simultanément ne permet pas d'ailleurs de préjuger de la nature de la liaison de $\mathrm{Cr}_{2} \mathrm{O}_{3}$ aux particules de digesta. Des études complénentaires sont nécessaires à cet égard et seront réalisées.

Il faut d'ailleurs remarquer avec MACRAE (r974) qu'il n'est pas toujours nécessaire que cette liaison soit très étroite pour que soient valides les résultats obtenus avec des indicateurs. Nous pensons notamment à la détermination de la quantité de fèces produites en vue de l'étude de la consommation à l'herbage ainsi qu'à la digestibilité des rations, domaines où la forme d'administration $\mathrm{du}^{\mathrm{Cr}} \mathrm{Cr}_{2} \mathrm{O}_{3}$ proposée contribuera sans doute à une amélioration de la qualité des résultats, du fait d'une grande régularité d'excrétion et d'une simplification des manipulations.

Pour l'étude quantitative des phénomènes de la digestion, cependant, on ne peut conclure des résultats enregistrés aux divers niveaux du tube digestif à une réhabilitation du $\mathrm{Cr}_{2} \mathrm{O}_{3}$ que MACRAE (I974), travaillant sur moutons, a proposé de remplacer par les radiolanthanides ou par le complexe ruthénium phénanthroline marqué au ${ }^{103} \mathrm{Ru}$ (TAN, Weston et Hogan, I97I) lors de l'emploi de canules en T. Dans ce type d'application, en effet, le transit simultané du marqueur et des digesta détermine la validité des résultats. Les contenus digestifs, particulièrement fluides dans le duodénum, obtenus, dans notre cas, en vidant complètement les divers segments, ont été homogénéisés avant analyse; dans ces conditions, le problème de la représentativité des échantillons ne se pose évidemment pas.

Il faut cependant noter qu'il n'est pas étonnant que le mode d'administration choisi par MACRAE et UlyaT' (I972) - une dose de $6 \mathrm{~g}$ de papier à l'oxyde de chrome administrée en une fois par une fistule du rumen - détermine dans la concentration de la phase solide en $\mathrm{Cr}_{2} \mathrm{O}_{3}$ des fluctuations plus importantes que celles observées pour le ${ }^{103} \mathrm{Ru}$, administré par perfusion continue du complexe par la fistule du rumen.

Quoi qu'il en soit, des essais complémentaires sont envisagés pour comparer le comportement de $\mathrm{Cr}_{2} \mathrm{O}_{3}$ à celui de ${ }^{144} \mathrm{Ce}$ dans l'intestin grêle, notamment, avec des animaux munis de fistules. 
En conclusion, l'étude comparée du transit de ces deux indicateurs nous a montré que, pour autant qu'il soit utilisé sous la forme de pellets au papier imprégné, le $\mathrm{Cr}_{2} \mathrm{O}_{s}$ peut avantageusement remplacer le ${ }^{144} \mathrm{Ce}$ dans certains cas ot̀ l'emploi de l'indicateur radioactif n'est pas autorisé ou souhaité : détermination de la quantité d'herbe ingérée en prairie et du coefficient de digestibilité des rations. Le mode d'administration mis au point pour ces essais permet d'administrer l'oxyde de chrome-papier haché et incorporé à des pellets, quantitativemient et sans difficulté. En outre, l'animal n'est ni blessé, ni perturbé, ce qui peut être le cas lors de l'emploi de la technique de TrOELSEN (Ig66), nécessitant l'utilisation d'un "balling gun ".

Accepté pour publication en avril 1978.

\section{Remerciements}

Lors de la préparation des pellets contenant l'oxyde de chrome-papier, nous avons bénéficié de l'aide du Groupe du Ministère de l'Agriculture pour l'étude de l' "Influence des traitements mécaniques et hydrothermiques sur la valeur nutritive des aliments pour bovins ". Dir. : Prof. M. VAN BELLE, Université Catholique de Louvain.

\section{Summary}

\section{Comparison of chromium sesquioxide-paper and radiocerium, particle markers of digesta, in sheep}

A comparative study of faecal excretion and gastrointestinal transit of radiocerium and chromium sesquioxide-paper in sheep showed that the behaviour of the two indicators is practically identical. The faecal recovery obtained for $\mathrm{Cr}_{2} \mathrm{O}_{3}$, virtually quantitative, confirms its often questioned unabsorbable character. The form of administration proposed for $\mathrm{Cr}_{2} \mathrm{O}_{3}$-paper - chopped, mixed with lucerne hay and pelleted with a die press - allows a quantitative ingestion without disturbing the animal. The method was applied to two groups of 3 sheep each. Radiocerium. was offered with the chopped meadow hay (totally and uniformly matked) to the first group of 3 sheep, with the lucerne pellets, incorporating the 2 indicators, to the second group. The results obtained widen the field of applications for chromium sesquioxide-paper administered in pellets.

\section{Références bibliographiques}

Clanton D. C., ig62. Variation in chromic oxide methods of determining digestibility of hand fed beef cattle rations. J. Anim. Sci., 21, 214-218.

Clawson J. T., Reid J. T., Sheiry B. E., Willman J. P., i955. Use of chromium oxide in digestion studies with swine. J. Anim. Sci., 14, 7oo.

Corbet' J. L., Greenitalgh J. F. D., Gwynn P. E., Walker D., i958. Excretion of chromium sesquioxide and polyethylene glycol by dairy cows. Br. J. Nutr., 12, $266-276$.

Corbett J. I., Greenhalgi J. F. D., McDonald I., Florence E., i96o. Excretion of chtomium sesquioxide administered as a component of paper to sheep. Br.J. Nutr., 14, 289-299.

CoWlishaw S. J., AldER F. E., i963. A comparative study of paper and oil as carriers of chro. mium sesquioxide administered to grazing steers to determine their faecal out prit. $J . B r$. Grassld. Soc., 18, 228-333. 
Crampton E. W., Lroyd L. E., I95r. Studies with sheep on the use of chromic oxide as an index of digestibility of ruminant rations. $J$. Nutr., 45, $3^{19-327}$.

Curran M. K., LEAver J. D., WeSTON F. W., I967. A note on the use of chromic oxide incorporated in a feed to estimate faecal output of ruminants. Anim. Prod., 9, 56I-564.

DAVIS C. L., BYER J. H., LUBER L. E., I958. An evaluation of the chromic oxide method of determining digestibility. J. Dairy Sci, 41, $15^{2-1} 59$.

Deinua B., Immink H. J., DEIJs W. B., I962. The excretion of chromium sesquioxide faeces by cows after administration of $\mathrm{C}_{2} \mathrm{O}_{3}$-containing paper. Jaarb. Inst. biol. scheik. Ondevz. LandbGewass, 188, I23-129.

EDIN H., I9I8. Orienterande Foraok over en pa hedkroppsprincipen. Grumded Metod att Bestamma en Foderbland nings Smalbarhet. Centralanst. foer Foersoeksv. pa Jordbruksomradet. (Stockholm) Meddedl., 165, I.

Elam C. J., Putnam P. A., Davis R. E., r959. Fecal excretion pattern of chromic oxide administered to Hereford heifers in a completely pelleted ration. J. Anim. Sci., 18, 718-725.

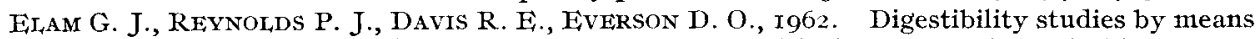
of chromic oxide, lignin and total collection techniques with sheep. J. Anim. Sci., 21, I89-192.

ELLIS W. C., I968. Dysprosium as an indigestible marker and its determination by radio-activation analysis. $J . A g r . F d$. Chem., 16, 220-224.

Fil,IS W. C., Huston J. E., 1968. ${ }^{144} \mathrm{Ce}-{ }^{144} \mathrm{Pr}$ as a particulate digesta flow marker in ruminants. J. Nutr., 95, 67-78.

François E., Compere R., Rondi G., Ig68. Fitude comparée de la vitesse de passage des aliments et des résidus alimentaires non digérés dans le tractus digestif du Rat et du Mouton. Bull. Rech. Agron. Gembloux, 3, 655-688.

Françors E., Compere R., r97I. Mesure du transit gastrointestinal chez le Mouton à l'aide des radiolanthanides. Bull. Rech. Agron., Gembloux, 4, 43-6I.

F'RANÇOIS E., COMPERE R., I972. Influence de la quantité ingérée sur le transit gastrointestinal et la digestibilité d'un foin de graminées long ou condensé chez le Mouton. In : Isotope studies on the physiology of domestic animals, 30I-3I9. International Atomic Energy Agency, Vienna.

François E., THEwis A., 1976. Le radiocérium utilisé comme élément de référence dans le tractus digestif du Mouton. Réalisation d'un marquage régulier. Bull. Rech. Agvon. Gembloux, $11(\mathrm{I}-2), 87-100$.

François E., TiIII. N., Thewis A., I978. Méthode rapide de dosage de l'oxyde de chrome dans les aliments, les fèces et les contenus digestifs par titrage après oxydation nitro-perchlorique. Ann. Zootech., 27, 355-36r.

Grovum W. L., Williams V. J., I973. Rate of passage of digesta in sheep. I. The effect of level of food intake on marker retention times along the small intestine and on apparent water absorption in the small and large intestines. Br. J. Nutr., 29, I3-2I.

Grovum W. L., Hecker J. F., r973. Rate of passage of digesta in sheep. II. The effect of level of food intake on digesta retention times and on water and electrolyte absorption in the large intestine. $B r . J . N u t r ., 30,22 \mathrm{I}-230$.

Hardison W. A., Linkous W. N., ENGEL R. W., I959. Observations on the use of $\mathrm{Cr}_{2} \mathrm{O}_{3} \mathrm{for}$ estimating the fecal output of dairy animals. $J$. Dairy Sci., 42, 346-352.

Huston J. E. et Erris W. C., I 968 . Fvaluation of Certain Properties of Radiocerium as an Indigestible Marker. J. Agv. Food Chim., 16, 225-230.

KANE E. A., JACOBSON W. C., MOORE L. A., I95\%. A study of the use of chromium oxide and lignin as indicators of digestibility. J. Dairy Sci., 33, 385-386.

KNAPKa J. J., BARTh K. M., BROWN D. G., CRAGLF, R. G., ig67. Evaluation of polyethylene, chromic oxide and cerium-I 44 as digestibility indicators in burros. $J$. Nutr., 92, 79-85

IAANGi,ands J. P., CorbetT J. L., McDonald I., REID G. W., I963. Estimation of faeces output of grazing animals from the concentration of chromium sesquioxide in a sample of faeces. I. Comparison of estimates from samples taken at fixed times of day with faeces outputs measured directly. Br. J. Nutr., 17, 2 I I-2 I 8.

I.ANGlands J. P., Corbiett J. L., McDonald I., REID G. W., I963. Estimation of faeces output of grazing animals from the concentration of chromium sesquioxide in a sample of faeces. II. Comparison of estimates from samples taken at fixed times of day with estimate from samples collected from the sward. Bv.J. Nutv., 17, 219-226.

LAssitfr J. W., Alitgood V., McGaughey G. H., i966. Chromic oxide as an indicator of digestibility of all-concentrate rations for sheep. J. Anim. Sci., 25, 44-47.

MACGUiRf, R. L., BRadiey N. W., LiTTLE C. O., ig66. Fiffects of frequency of feeding on excretion of chromic oxide, crude protein and gross energy on nutrient digestibility by steers. J. Anim. Sci., 25, I85-19г. 
Macrae J. C., UlyatT M. J., 1972. Comparison of spot and continuous sampling for estimating digesta flow in sheep. N.Z.J.Agr. Res., 15, 98-106.

MACRAE J. C., I974. The use of intestinal markers to measure digestive function in ruminants. Proc. Nutr. Soc., 33, I47-I54.

MoORE, J. H., r957. Diurnal variation in the composition of the faeces of pigs on diets containing chromium oxide. Br. J. Nutr., 11, 273-288.

PETRY H., EndERs H., I974. Kritische Betrachtungen über die Zuverlässigkeit der in klassischer Verdauungsversuch und nach der Chromoxid - Indikatormethode bestimmten Verdauungskoeffizienten. Z. Tierphysiol. Tievernähv. Futtermittelk., 33, 88-98.

Phar P. A., Bradiey N. W., LitTle C. O., CundifF L. V., r97o. Effects of confinement and level of feed intake on digestibility of nutrients and excretion of chromic oxide, crude protein and gross energy in the bovine. J. Anim. Sci., 30, 589-592.

Poncerr C., r976. Utilisation du Cérium-I4 I comme marqueur de la phase solide des contents digestifs chez le ruminant. II. Comportement du marqueur au niveau du duodénum et au niveau des fèces. Ann. Biol. anim. Bioch. Biophys., 16, 74I-75I.

Schurch A. F., Llovd L. E., Crampton E. W., I950. The use of chromic oxide as an index for determining the digestibility of a diet. $J$. Nutr., 41, 629 .

Tan T. N., Weston R. H., Hogan J. P., I97I. Use of ${ }^{103}$ Ru - labelled tris (I, ro-phenanthroline) Ruthenium (II) Chloride as a Marker in Digestion Studies with Sheep. Int. J. Appl. Rad. Isot., 22, $301-308$.

Thewis A., François E., Thiı, N., I975. Le transit gastrointestinal chez le Ruminant mesuré à l'aide des radiolanthanides. Signification des paramètres de la phase exponentielle des courbes de concentration fécale en traceur. Bull. Rech. Agron. Gembloux, 10, 307-326.

Thewis A., François E., Debouche C., Thielfemans M. F., i976. Utilisation des radiolanthanides dans la détermination du transit gastrointestinal chez les petits ruminants. Comparaison des techniques directe (abattage) et indirecte. Ann. Zootech., 25, 373-385.

TROELSEN J. E., I966. Pelleting of chromic oxide paper for administration to cattle and sheep. Can. J. Anim. Sci., 46, 226-227

UThey P. R., Bol.iNg J. A., Bradley N. W., Tucker R. E., I97o. Recovery of radioactive chromic oxide from the bovine gastrointestinal tract. $J$. Nutr., 100, I227-I 232 .

VON ENGEIHARDT W., I974. Radioactive reference substances in gastrointestinal studies. Tracer techniques in tropical animal production. I.A.E.A., Vienna, I I I-I24.

Verite R., Poncet C., Chabi S., PIon R., i977. Utilisation des tourteaux traités au formol par les vaches laitières. I. Aspects digestifs. Ann. Zootech., 26, I67-I 8 I. 